CASE REPORT

\title{
Observations of Thoracic Neuromuscular Oscillation Subsequent to Thoracic Pathology
}

\author{
Lysander $\mathrm{Jim}^{1 *}$ and Stuart McGill ${ }^{2}$ \\ ${ }^{1}$ Mastery Medical Center, South Pasadena, CA, USA \\ ${ }^{2}$ Department of Kinesiology, University of Waterloo, Waterloo, Ontario, Canada
}

\begin{abstract}
In this paper we propose that a tremor occurring over a thoracic myotomal region as representative of a motor manifestation of thoracic radicular injury. We term this process thoracic neuromuscular oscillation. It is characterized by synchronous firing of the unilateral oblique muscles at one or more adjacent thoracic myotomal distributions at a frequency of 8 to $10 \mathrm{~Hz}$.

Six cases of observed regular continuous abdominal wall muscular tremor occurring after traumatic thoracic spine pathology are presented. These cases were drawn retrospectively from patient files obtained over 20 years. We refer to this tremor, measured to be $8-10 \mathrm{~Hz}$, as thoracic neuromuscular oscillation. The neuromuscular oscillation was influenced by mechanical forces, specifically spine loading in either a bending or compressive mode. In some cases, cervical position modulated the tremor. Other examples of thoracic spine pathology that occurred comorbid to thoracic neuromuscular oscillation are noted.
\end{abstract}

\section{Keywords}

Open Access

\section{Citation: Jim L, McGill} S.Observations of Thoracic Neuromuscular Oscillation Subsequent to Thoracic Pathology. Physical Medicine and Rehabilitation. 2018; 2(1): 2.

Received: June 06, 2018 Accepted: November 26, 2018 Published: December 27, 2018

Copyright: $\odot 2018 \mathrm{Jim}$ L. This is an open access article distributed under the terms of the Creative Commons Attribution License, which permits unrestricted use, distribution, and reproduction in any medium, provided the original author and source are credited.

Corresponding author: Lysander Jim, MD Mastery Medical Center South Pasadena, California Email: lysanderjim@gmail.com
Thoracic spine injury, neuromuscular oscillation, thoracic myotome, thoracic pain

\section{Introduction}

Myotomal manifestations of thoracic radicular injury remain largely unreported in the medical literature. Descriptions of thoracic radicular injury are generally framed within the context of dermatomal sensations of pain, numbness, paresthesias and dysesthesias. ${ }^{1}$ By contrast, in the literature on cervical and lumbar radicular nerve injury, the motor impairment of weakness through predictable myotomal patterns is widely known. It is possible that the absence of clinical approaches for motor testing of individual thoracic myotomal levels explains the dearth of myotomal correlates for thoracic radicular injury.

In this paper we propose that a tremor occurring over a thoracic myotomal region as representing a motor manifestation of thoracic radicular injury. We term this process thoracic neuromuscular oscillation. It is characterized by synchronous firing of the unilateral oblique muscles at one or more adjacent thoracic myotomal distributions at a frequency of 8 to $10 \mathrm{~Hz}$. A more extensive variant involving the entire abdominal wall at the same frequency is also described. Notably, whether strictly following a thoracic myotome or involving the entire abdominal wall, this tremor is almost entirely reproducible and relieved by alterations of mechanical loading. Thoracic dermatomal pain, paresthesias or dysesthesias are present in some but not all cases.

We present six cases where this tremor occurred, all of which occurred in people with mid to lower thoracic injury ranging from T6 to T12.

The genesis for this paper came from a discussion between the authors of their challenging patients who displayed abdominal wall muscular tremor when they came to the realization that the common feature of all patients in question was documented thoracic spine trauma. Both 
authors are spine specialists with approximately 40 years of clinical observations. SM has seen six similar cases over his career while LJ has seen one over this period. At the time of patient appointments, we did not realize, nor understand, what we were seeing. However, we were motivated to go back through our patient files retrospectively and recover whatever data we could, albeit in some cases quite thin.

Since this case study report represents the first known description in the scientific literature of a thoracic neuromuscular oscillatory phenomenon occurring with predictable mechanical forces at this particular frequency, there are no reliable estimates on the prevalence of this type of tremor. Based on observation of the patients in this sample, it is a significant source of morbidity to the subgroup of spine patients affected by it. One report identified lateral abdominal wall paresis with low thoracic level disc bulges and neural compression, but there was no mention of any associated tremor. ${ }^{2}$

Thoracic neuromuscular oscillation can impair quality of life through its effects on pain, function and social stigma. Medical misdiagnosis was universal among the patients referred to us because of lack of recognition of the mechanisms. The tremor aspect has been mistaken for other categories of movement disorders such as epilepsy, upper motor neuron dysfunction, spinal cord injury and neuromuscular disease. More commonly, the symptoms are assumed to lack a pathoanatomic basis and this phenomenon is mislabeled as malingering, psychosomatism and pseudoseizure. These misinterpretations belie the pathophysiological rationale for thoracic neuromuscular oscillation and the possibility of effective treatment to mitigate its effects.

The purpose of this report is to establish the clinical features of thoracic neuromuscular oscillation from the review of six case studies. It includes the first electrodiagnostic data that we are aware of published on thoracic neuromuscular oscillation and offers perspectives on an effective treatment for it. As the patients presented to the clinic we had no idea that an eventual subgroup would form, compromising the rigor in terms of variable and test protocol control. This is common with the emergence of rarer conditions that trouble and frustrate clinicians. Collectively, these cases suggest a possible definition of neuromuscular oscillation together with a possible pathophysiological explanation.

\section{Methods}

Author SM specializes in investigating challenging back pain cases. A few patients over a span of 30 years presented with a distal muscular tremor specifically in the rectus abdominis and/or the oblique muscles. The frequency of synchronous bursts was confirmed with electromyography to be approximately $8-10 \mathrm{~Hz}$. The common feature of these patients was a history of thoracic spine trauma. Author LJ noted a similar pattern of neuromuscular oscillation with thoracic trauma and conferred with SM motivating this manuscript. Subsequently, patient files were reviewed and relevant data recovered and described. Resonant pulses were only present when the spine was mechanically bent or compressed. Compressive load can result from muscle activation in the torso. Thus a description of patient presentation features follows.

\section{Description of injury}

There a total of six case studies presented in this paper. The first patient had a compression fracture at T9 from a motorcycle accident. The second patient injured T12 and L1 after a fall. The third patient had a crush injury of the spine. The fourth patient had a fall leading to T6 fracture. The fifth patient had a T10 fracture aftera fall. The sixth patient was a forester who had a T6-T7 and T7-T8 injury after getting hit by a falling tree.

\section{Study Design}

Aconvenience sample put together retrospectively from patient records.

\section{Results}

\section{Case Study 1}

A 35 years old male suffered a T9 compression fracture subsequent to a motorcycle accident. In the accident, the patient was thrown from his motorcycle and landed on his back onto the ground. In the emergency room later that day, CT imaging without contrast revealed a T9 compression fracture. About two weeks after his accident, the patient noticed that he had a tremor on his right oblique muscles in a narrow strip above his umbilicus at unpredictable 
times. This tremor would progress to painful spasms of the oscillating region of his right oblique. He identified provoking activities to include prolonged sitting, driving, sex, sit-ups, jujitsu (which he had to quit) and sneezing. The patient did not understand why he would have these tremors and saw multiple doctors who were unable to provide any diagnosis, but ruled out other forms of tremor including thyrotoxicosis, drug-induced tremor (the patient was not on any drugs), and essential tremor. Parkinsonian tremor was also ruled out on the basis of clinical features. On initial evaluation with one of the authors, LJ, the patient demonstrated a regular, continuous, tremor over the T9 dermatomal/myotomal region of the right oblique. The patient did not have tremor in any other region such as the limbs or the left obliques. He had preserved sensation of his entire trunk and limbs. His strength examination of the upper and lower extremities was normal. He did not have upper motor neuron signs such as Hoffmans, ankle clonus or increased upper or lower limb tendon reflexes. This tremor could be consistently provoked with prolonged thoracolumbar flexion and flexion with high compressive loads. On follow-up over the next several months, the patient decreased the frequency of these episodes, by reducing the frequency of prolonged compression and compressive loads.

\section{Case Study 2}

A 53 years old female, seen in May, 2011, suffered cervical trauma from a fall of almost 4 meters. Her fall caused trauma to her cervical spine and x-rays of the spine revealed compression fractures at T12 and L1. Within several months, she reported the development of a "weird feeling" (her description) and tremor contractions on the abdominal wall. Magnetic resonance imaging of the thoracic spine without contrast revealed posterior-central disc herniations at the T5-6, T7-8, and T9-10 levels. A spondylolisthesis was also observed at the T6-7 level together with narrowed disc spaces at the T5 to T10 levels. Nerve conduction tests found that the peroneal nerves and right lower extremity were normal. Provocative testing revealed she was flexion intolerant since spine flexion caused shaking (8 Hz tremor) due to synchronous abdominal oblique wall contraction. The Standing Drop test 20 superscripted resulted in pain when the abdominals were voluntarily relaxed, but there was no pain when braced with stiffening contraction of the abdominal wall and latissimus dorsi muscles. During the prone instability test of shear load tolerance, the apex of pain sensitivity was at the T12 level. Standing extension created pain in a unilateral T12 distribution. During spine flexion and extension in the quadruped position, there appeared to be a spinal hinge at the T12 level. It was concluded that neither compression nor bracing caused the neuromuscular oscillation but bending the joint triggers pain and tremor of $8 \mathrm{~Hz}$ in the unilateral abdominal oblique wall (measured at the time of clinic evaluation and noted but for which EMG records were not retained). As this was an early patient we did not realize the significance of these observations at the time. Practicing the principles of spine hygiene to shift torso flexion from the spine to the bending about the hips reduced her symptoms substantially thus assisting in the diagnostic convergence of the mechanism involving a dynamic disc bulge on the root(s) serving the abdominal wall. ${ }^{3}$

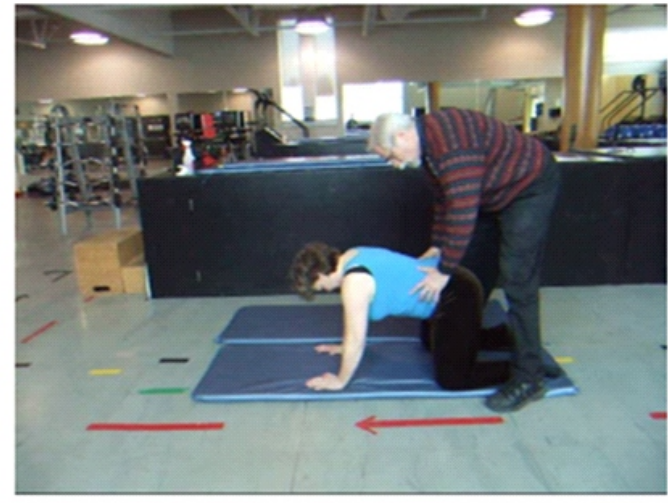

Figure 1. This screen capture of a video showed the abdominal wall tremor with spine flexion. Specifically, when all fours she flexed her spine "humping up like a camel". When asked what she knew about the response, she replied, "I do not know what this shaking is but it happens every time I bend my spine forwards like this." 


\section{Case Study 3}

A22-year-old male was seated in his car as a feed truck rolled over and crushed down his car roof and imposing a crush load on his spine in July of 2007. Clinical observation by the author confirmed his medical reports of an altered movement pattern for a sit-to-stand transition that had eluded characterization with prior specialists including physiatrists and neurologists. This movement pattern manifested as a bizarre and persistent ritual for rising from a chair that consisted of leaning forward slightly, punching one fist up in the air and then standing stiffly. The patient claimed that he felt compelled to perform these movements and said his movements were "self- found" and noted that "they work".

The report from a University Rehabilitation institute documented the use of surface EMG to record muscle patterns that were compared with population norms. Notable features included: loss of balance when standing with closed eyes (positive Romberg sign); stress to his spine resulted in a tremor in the abdominal obliques, specifically a $8-9 \mathrm{~Hz}$ shaking. This neuromuscular oscillation was seen also in the tibialis anterior with flexion that occurred with bending his thoracic spine to pick up a light object from the floor. This was repeatable over three trials.

\section{Case Study 4}

A 55-year-old female fell at home causing a fracture of T6 in Sept of 2001. Clinical assessment revealed that when standing, spine extension or lateral bending caused torso oscillations which in turn caused her loss of balance. We also noted that tiling her head modulated the amplitude of the muscle oscillations. Our recent work has shown changes in neural dynamics along the cord with cervical flexion and rotation. ${ }^{4}$

\section{Analysis}

At the time we considered the mechanism of varying amplitude of the muscle oscillation to be vestibular and referred her to an ortolaryngologist. In hindsight, and after better understanding this pattern of thoracic trauma linked to oscillation, we now would have considered that the repositioning of the head changed neural tension through the thoracic spine.

Specifically, we have found that cervical flexion, lateral bending and twisting creates unique tensions in the descending spinal cord. To be more precise, our work documented several amended neural outputs during different head-neck positions. For example, axial head rotation decreased neural conduction velocity in opposite arm ulnar nerve and increased proximal motor ulnar mean latency when compared with a neutral cervical head - neck position due to changes in neural tension. The median nerve was not influenced in this way probably due to anatomical differences and its pathway under the ulnar groove of the elbow joint.

\section{Case Study 5}

A 58-year-old female fractured T10 following a motor vehicle accident in 2007. The patient had normally preserved strength, intact sensory exam to light touch and preserved strength of the limbs and torso. During the consulting exam for back pain she mentioned the loss of the ability for simple movement patterns such as crossing her legs when seated. On examination, the patient demonstrated the neurological capacity to perform each of the individual actions required to sit and cross her legs, but was unable to execute the sequence of movements needed to complete the action. On Magnetic Resonance Imaging of the thoracic spine, we also noted the presence of multiple "black dots" appearing on the cross- sectional image of the spinal cord at the T10 level. Once again, at the time of examination, we did know to make better documentation other than noting this in our clinical notes, specifically that these features were unique to the T10 level. When spine flexion was performed, oblique abdominal wall muscle contraction tremor occurred with a frequency of $8-10 \mathrm{~Hz}$. Consultation with neurology failed to identify the source of this isolated region of tremor which did not affect her limbs.

\section{Analysis}

Along with the tremor, the onset of a separate movement impairment in sequencing appeared to be a case of corrupted motor engrams in the mid thoracic levels. Despite a normal strength and sensory exam, the patient had "forgotten" how to cross her legs and slowly regained the ability only after training the movement in chunks. Spinal cord injuries are associated with alterations of motor tasks. ${ }^{5}$ It may be the patterned engram resided in the cord 
at the damaged level (level of fracture and appearance of the cord cross-section). Engrams are encoded motor maps that drive a movement pattern. ${ }^{6}$ Latash (2005) discusses several associated organs along descending pathways in the spinal cord that could corrupt the engram. ${ }^{7}$ Disrupted engrams could challenge the execution of simple movements. Of note, the presence of altered movement sequencing and patterns (corrupted motor engrams) is distinct from thoracic neuromuscular oscillation. Corrupted motor engrams represents a central nervous system injury ${ }^{7}$ whereas thoracic neuromuscular oscillation probably represents a peripheral nervous system injury. ${ }^{8}$ A fuller discussion of the pathophysiology of thoracic neuromuscular oscillation is provided in the discussion.

\section{Case Study 6}

A 40-year-old male working as a forester was hit by a log in December of 2000. On urgent medical evaluation, an x-ray of the thoracic spine demonstrated a T9 fracture. Magnetic resonance imaging of the thoracic spine without contrast revealed posterior disc bulges at T6-7 and T7-8. The patient reported experiencing different kinds of muscle "spasming" depending upon the task he performed that were linked to his back pain. Examination revealed that spine flexion caused neuromuscular oscillation in his abdominal wall muscles at the frequency of approximately $10 \mathrm{~Hz}$ (see figure 2).

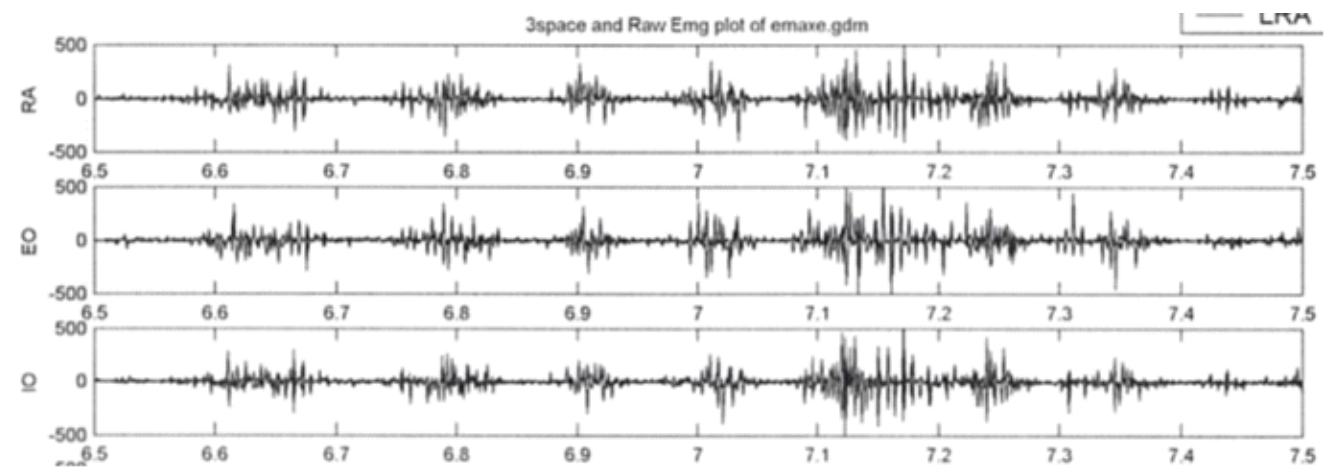

Figure 2. Case study 6: During the Biering-Sorensen task (the upper torso is cantilevered out over the end of a test table with the feet restrained so that the muscles must hold the full upper body bending load) used for EMG normalization synchronous firing of motor units, all torso muscles engaged in a tremor response (rectus abdominis, external oblique, internal oblique). Note that the graphics display normalizes amplitude to the largest observed in that muscle over the 2 second collection period. Unnormalized amplitudes would demonstrate the much higher real amplitudes observed in the extensor muscles needed to support the cantilevered prone upper body.

As the effort to flex increased, the low back extensor muscles joined in in the resonant tremor in an antiphase pattern. Furthermore, when spine load from torso muscle contraction was increased, together with spine bending, a stronger tremor resulted. However, spine load as a single triggering variable was confirmed during gait testing. Left heel strike during walking caused the abdominal muscle tremor. This response was increased if the subject was sitting first and then transitioned to standing, then walking. Cycling on a stationary bike did not cause this response - we assume because the spine load was of insufficient magnitude. The largest amplitude of abdominal tremor created during testing occurred during the Biering-Sorensen extensor hold exercise. Extensor muscle tremor was very little. This exercise creates approximately $2800 \mathrm{~N}$, on average, of compressive load on the spine. ${ }^{9}$

Two neurologists were consulted but neither had any thoughts to offer other than they had not seen such a response before although they admitted they were not used to seeing muscle EMG over the time history of a task. Both neurology consultations could not attribute this patient's tremor to any known metabolic or neurological cause of tremor.

\section{Analysis}

In explaining the extensor muscle contraction anti-phase oscillations, we do not know if the extensor involvement was due to more pressure on the spine and thus discs, or if this was an anti-phasic mechanical response to the flexor pulses to maintain torso balance. This former explanation is consistent with a dynamic disc bulge that increases pressure on neural tissues during the spine flexion bending associated with sitting. ${ }^{10,11}$ 


\section{Discussion}

\section{Features of thoracic neuromuscular oscillation}

We are identifying thoracic neuromuscular oscillation of the oblique muscles as a newly identified motor manifestation of thoracic spine injury. Specifically, it appears most likely associated with axonal irritation of the unilateral or bilateral thoracic radicular motor neuron since the roots that serve rectus abdominis and the oblique muscles are associated with $\mathrm{T} 9$, T10, T11. The extent of thoracic nerve root involvement is a probable pathophysiological explanation for the distribution of tremor. In our sample, the most common provocative factors of neuromuscular oscillation are thoracic spine flexion (bending) and compression loads. Initiation and perpetuation of these postures and loads causes irritation of motor axon(s), leading to spontaneous discharge of one or more motor units at the characteristic frequency of motor unit discharge of $6-10 \mathrm{~Hz} .{ }^{12}$ Conversely, removal of these triggering forces caused reduction followed by cessation of the tremor.

Thoracic neuromuscular oscillation is more likely related to peripheral rather than central nervous system injury. While there was evidence of spinal cord injury in some of the cases, notably cases 2,3 and 5 (who had spinal cord changes on MRI at the thoracic level of interest), most of the patient cases did not have documented clinical or imaging evidence of central nervous system injury. There are other features that suggest that neuromuscular oscillation is caused by a peripheral rather than central lesion. First, this movement disorder is anatomically correlated with a peripheral site of injury; for example, in case 1 , the patient had a T9 fracture and oscillation localized to a unilateral T9 dermatomal/myotomal distribution. Also, the tremor frequency falls within the known established range for motor unit discharge rather than the known resonance frequencies for spinal oscillations.

As such, classification of thoracic neuromuscular oscillation would fit within the established classification of peripherally induced movement disorders. Our cases match a proposed clinical criteria for movement disorder previously described for peripherally induced movement disorders (PIMD): 1) the trauma is severe enough to cause local symptoms for at least 2 weeks or requires medical evaluation within 2 weeks after trauma, 2) the initial manifestation of the movement disorder is anatomically related to the site of injury; and 3) the onset of the movement disorder is within days or months after the injury. ${ }^{13}$ While there are no prior case studies of PIMD related to thoracic radicular injury, there was one case of neuromuscular oscillation related to cervical radicular injury. ${ }^{14}$ Tellingly, this patient had a unilateral right hand tremor at a 6 to 8 Hertz frequency, a characteristic frequency for motor unit discharge.

Furthermore, thoracic neuromuscular oscillation is inconsistent with classification as a spinal or peripheral myotonia. Spinal myoclonus is rare, involves groups of muscle supplied by a few continuous segments of the spinal cord, is rhythmic, bilateral and slow (under four $\mathrm{Hz}){ }^{15}$ By contrast, thoracic neuromuscular oscillation involves only a part of the oblique (rather than groups of muscles), is sometimes unilateral and faster than $4 \mathrm{~Hz}$. Thoracic neuromuscular oscillation is also distinct from radicular myoclonus which has been characterized as rhythmic, $100-200 \mathrm{~ms}$ in duration and at $4 \mathrm{~Hz} .{ }^{16}$ While rhythmic, thoracic neuromuscular oscillation lasts for several seconds and has a higher frequency.

Likewise, thoracic neuromuscular oscillation should not be classified as a myokymia. The mechanism of myokymia is characterized by brief bursts of single motor unit potentials recurring at regular or irregular intervals. ${ }^{17}$ Myokymia can cause regular or irregular discharge of groups of motor units. It is continuous undulation of a group of muscle fibers caused by the successive spontaneous contraction of motor units. On EMG, they appear as groups of 2 to 10 potentials, fire at $5-60 \mathrm{~Hz}$ and recur regularly at 0.2 to 1 second intervals. By contrast, thoracic neuromuscular oscillation is not spontaneous (having predictable mechanical provocations), is not undulating, has a narrower and generally lower frequency range and does not recur at short intervals. It is worth noting that the site of pathology, the motor axon, is shared between myokymia and thoracic neuromuscular oscillation, but not the mechanism of injury. In myokymia, the unstable axon membrane creates spontaneous ectopic discharges. In thoracic neuromuscular oscillation, mechanical irritation of an otherwise generally stable motor axon stimulates discharge.

In summary, thoracic neuromuscular oscillation is most accurately classified as a peripherally induced movement disorder distinct from myotonia and myokymia. The 
pathophysiology is mechanical irritation of the thoracic motor axon, leading to a characteristic 6 to $10 \mathrm{~Hz}$, long, rhythmic, continuous, unilateral or bilateral oblique tremor within a thoracic myotomal/dermatomal distribution. The mechanical stress on the nerve may be due to a disc bulge/herniation or other anatomic vulnerability such as that seen following thoracic vertebral fracture. The tremor may be painless, painful immediately or painful after several cycles of tremor. Pressure change in neural tissues linked to spine curve has been documented by Shacklock. ${ }^{18}$ Muscle pain is also possible from sustained contractions (spasm) leading to local hypoxia of the muscle. In some, neuromuscular oscillation may lead to a secondary generalized tremor, likely a form of clonus. This generalized tremor is distinct from thoracic neuromuscular oscillation and present only in a subset of patients with thoracic neuromuscular oscillation.

\section{Clinical Diagnostic Features}

Thoracic neuromuscular oscillation of the mid and thoracic spine consists of tremor in the affected myotome or more extensively along the abdominal wall. The most common triggers are spine flexion and compression, thus spine load. Accuracy of diagnosis is established by the ability to reproduce neuromuscular oscillation through mechanical positioning of the thoracolumbar spine. Positional adjustments of the cervical spine may modulate the frequency or the intensity of the tremor. Most likely, cervical position influences tension of the spinal cord and thoracic spinal nerves Shacklock provides a variety of examples. ${ }^{19}$ Electrodiagnostic study (surface EMG) would also corroborate this diagnosis based on the finding of the characteristic 8 to $10 \mathrm{~Hz}$ frequency of tremor.

\section{Treatment}

Mechanical variables are fundamentally important to provocation and persistence of neuromuscular oscillation. In each case study presented here, the magnitude of symptoms was modulated by spine bend or compressive load. Thus the treatment of neuromuscular oscillation is avoidance of provoking stresses to the thoracic radicular nerves. The patients reported here had reduced symptoms with avoidance of the mechanical trigger except case study 6 who reported only very minor reductions. We refer to this involved education on postures and movements together with skilled movement competency to reduce the offending variable as the practice of "spine hygiene". ${ }^{3}$ Specific examples include abdominal and thoracic muscle bracing with the magnitude tuned for optimal symptom relief, "hip hinging" movements to reduce spine bending and using movement strategies such as the golfer's lift and single-leg lunge to reach items on the floor. Education on sneezing with the head upturned to avoid thoracic compression with flexion was a helpful detail for some patients. Avoiding the trigger appeared to allow some desensitization to occur with varying success.

While spine flexion was the most common trigger, there were others given the variables that influence neural tension and ability to transmit pulse trains. ${ }^{4}$ In some patients, one in particular, spine extension was the trigger. This seemingly paradoxical move can tension a thoracic spine section of the cord of a nerve root that is "under hooked" by an offending disc bulge. ${ }^{20}$ We suggest that when performing diagnostic probing of the tremor trigger consider the least harmful and physiologically stressful tests. One example is the cat-camel movement, an exercise that allows for testing flexion and extension of the spine in a low compression state. Then moving to upright postures such as sitting and standing allow the addition of compressive load to spine motion. In summary, converging on an appropriate treatment of neuromuscular oscillation depends on first identifying the provoking trigger and training the requisite postures, motions and loads to avoid mechanical irritation of the thoracic radicular nerves.

\section{Conclusion}

In conclusion, compromise of the spinal cord and spinal nerves in the mid- and lower thoracic spine appears to be a common link in patients presenting with tremor-like neural responses that we have called thoracic neuromuscular oscillation. In every case the patient was labeled as bizarre or given non-specific categorization such as psychosomatism or fibromyalgia. We suggest that compromise of the central cord can account for thoracic neuromuscular oscillation observed in patients uniquely clustered due to mechanical damage to the lower thoracic spine and absent of other conditions or disease that could explain such observations. 


\section{References}

1. O'Connor RC, Andary MT, Russo RB, DeLano M. Thoracic radiculopathy. Phys Med Rehabil Clin NAm. 2002; 13(3): 623-644.

2. Stetkarova I, Chrobok J, Ehler E, Kofler M. Segmental abdominal wall paresis caused by lateral low thoracic disc herniation. Spine. 2007; 32(22): E635-E639.

3. McGill SM. Back Mechanic: The step-by-step McGill method to manage back pain. Backfitprolnc, 2015.

4. Petrovacki-Dejanovic B, DejanovicA, McGill SM. (in press), Head-neck posture influences motor nerve conduction velocity and latency in the upper extremity.

5. Edgerton VR, Leon RD, Harkema SJ, et al Retraining the injured spinal cord. J Physiol, 2001;533(1): 15-22.

6. Monfils MH, Plautz EJ, Kleim JA. In search of the motor engram: motor map plasticity as a mechanism for encoding motor experience. Neuroscientist. 2005;11(5): 471-483.

7. Adkins DL, Boychuk J, Remple MS, Kleim JA. Motor training induces experience-specific patterns of plasticity across motor cortex and spinal cord. J Appl Physiol. 2006;101: 1776-1782.

8. Latash M. Neurophysiologic basis of movement. ( $2^{\text {nd }}$ edn.), Human Kinetics Publishers, Champaign, IL, USA, 2008.

9. Kavcic N, Grenier SG, McGill SM. Quantifying tissue loads and spine stability while performing commonly prescribed low back stabilization exercises. Spine. 2004;29(20): 2319-2329.

10. Tampier C, Drake J, Callaghan J, McGill SM. Progressive disc herniation: An investigation of the mechanism using radiologic, histochemical and microscopic dissection techniques. Spine. 2007;32(25): 2869-2874.

11. Veres S, Robertson P, Broom N. The Morphology of Acute Disc Herniation: A Clinically Relevant Model Defining the Role of Flexion. Spine. 2009;34(21): 2288-2296.

12. Mills KR. The basics of electromyography. J Neurol Neurosurg Psychiatry. 2005;76(suppl 2): ii32-ii35.

13. Chung EJ, Kim SJ, Lee WY, et al. Four cases with peripheral trauma induced involuntary movements. $J$ Mov Disord. 2010;3(2): 39-41.

14. Ellis SJ. Tremor and other movement disorders after whiplash type injuries. $J$ Neurol Neurosurg Psychiatry. 1997; 63(1): 110-112

15. Campos CR, Limongi JC, Machado FC, Brotto MW. A case of primary spinal myoclonus: clinical presentation and possible mechanisms involved. Arq Neuropsiquiatr. 2003;61(1): 112-114.

16. Miki $\mathrm{Y}$, Tomiyama M, Ueno $\mathrm{T}$, et al. Radicular myoclonus in a patient with Guillain-Barré Syndrome. Intern Med. 2012;51(15): 2021-2023.

17. Gutmann L, Gutmann L. Myokymia and neuromyotonia 2004. J Neurol. 2004;251(2):138-142.

18. Shacklock M. Clinical Neurodynamics: a new system of musculoskeletal treatment. Elsevier, Oxford, 2005.

19. Shacklock M. Biomechanics of the Nervous System: Breig revisited. NDS Neurodynamic Solutions. Adelaide, 2007.

20. McGill SM. Low back disorders: Evidence based prevention and rehabilitation. ( $3^{\text {rd }}$ edn.), Human Kinetics Publishers, Champaign, IL, USA, 2016. 